\title{
Moscheen und Kulturvereine als Basisstationen Sozialer Arbeit
}

\section{Das Soziale und die islamischen Organisationen in Deutschland}

\author{
Gazi Caglar
}

Prof. Dr. Gazi Caglar ist Politik-, Geschichts- und Religionswissenschaftler und Hochschullehrer an der Fakultät für Soziale Arbeit und Gesundheit der Hochschule für angewandte Wissenschaft und Kunst in Hildesheim.

E-Mail caglar@hawk-hhg.de
Von Armut und Ausgrenzung sind Migrantinnen und Migranten, und damit auch Menschen muslimischen Glaubens, besonders betroffen. Die Zentralverbände der Muslime in Deutschland haben auf diese Herausforderung noch kaum reagiert. Dagegen erfüllen die örtlichen Moscheen und alevitischen Kulturvereine neben religiösen und kulturellen oft auch soziale Aufgaben gegenüber ibren Gläubigen.

Das Soziale gewinnt in den europäischen Gesellschaften wieder an Bedeutung. Haben mit den neuen sozialen Bewegungen nach 1968 Themen wie Naturschutz, Atomkraft, Kriegsgefahr, sexuelle Identität die Debatten bestimmt, rückt die soziale Frage angesichts neoliberaler Umstrukturierung gesellschaftlichen, ökonomischen, staatlichen und kulturellen Lebens in den Vordergrund. Die zunehmende Armut, die strukturelle Arbeitslosigkeit, die soziale Isolierung und Marginalisierung von immer größer werdenden Bevölkerungsgruppen, die Deregulierung der Lohnstruktur für Arbeitende, die Umorganisierung und zunehmende Privatisierung sozialer Dienstleistungen im Zeichen von sozialer Kälte haben die Krise der Arbeitsgesellschaft und die damit zusammenhängende soziale Dimension gesellschaftlicher Konflikte von neuem ins öffentliche Bewusstsein gerückt. Von der sozialen Umstrukturierung sind migrantische und damit muslimische Lebenslagen in besonderem Ausmaß betroffen.

\section{Die soziale Lage der Muslime in Deutschland}

Zwischen zwei Polen pendeln die gegenwärtigen Diskurse über Migration in Deutschland: die Skandalisierung der sogenannten Parallelgesellschaften und der Würdigung erfolgreicher Selbstständiger und einzelner Persönlichkeiten als Beispiele gelungener Integration. Doch jenseits der Rede von Normalisierung und Entspannung im Zeichen der Integration sind die sozialen Lebenslagen von Migrantinnen und Migranten in Deutschland und damit auch die der großen Mehrheit der Muslime von sozioökonomischer Marginalisierung und Armut bestimmt (vgl. Kasten »Vor Statistiken wird gewarnt ", Seite 124). Der Mikrozensus, die Arbeitsmarktstatistiken und die Daten des Sozio-Ökonomischen Panels sprechen eine eindeutige Sprache:

Den größten Anteil unter den ausländischen Erwerbstätigen stellen die angelernten Arbeiter mit 30,3 Prozent; bei den Deutschen sind es die mittleren und gehobenen Angestellten mit 42,2 Prozent. Der Beamtenstatus ist nach wie vor die rein gehaltene Domäne der Deutschen. Ausländer arbeiten auch 50 Jahre nach den ersten Anwerbeabkommen hauptsächlich im produzierenden Gewerbe. In Dienstleistungsbranchen sind sie unterrepräsentiert. Dies trifft auch auf die jüngeren ausländischen Erwerbstätigen in der zweiten und dritten Generation zu. Die Selbstständigenquote liegt bei den Deutschen bei circa 11,0 Prozent, bei den Migranten insgesamt bei 7,8 Prozent und bei den Türken und Kurden sogar bei nur 5,0 Prozent. Der Großteil dieser Selbstständigeninitiativen sind Klein- und Kleinstunternehmen, in denen weniger als drei Personen beschäftigt sind. Derartige Unternehmensgründungen sind vielfach Resultat der Chancenlosigkeit auf dem Arbeitsmarkt und eine Flucht in die Scheinselbstständigkeit.

Die Überrepräsentanz in den krisenanfälligen Branchen des produzierenden Gewerbes wirkt sich strukturell negativ auf das Einkommen der Migrantenfamilien aus. Personen in ausländischen Haushalten sind in viel stärkerem Maße als Deutsche von Niedrigeinkommen und Einkommensarmut betroffen. Die deutschen Haushalte verfügen monatlich im Durchschnitt über mehr als 600 Euro mehr als die ausländischen. Von 1996 bis in die 
Gegenwart hat sich die Einkommenssituation ausländischer Haushalte noch einmal verschlechtert.

Die Arbeitslosenquote ist unter Ausländern mit 20,4 Prozent weiterhin deutlich höher als die der Gesamtbevölkerung. In manchen Städten erreicht die Quote 35 Prozent. Das Armutsrisiko von ausländischen Personen ist von 1998 an weiter hochgeklettert. Die Sozialhilfequote ist mehr als doppelt so hoch wie bei den Deutschen.

Die sogenannte Bildungsarmut, die aufgrund des strukturell selektiven Charakters der Schule systematisch reproduziert wird, hat inzwischen Eingang in die UNBerichte über Deutschland gefunden.

Der vorherrschende wissenschaftliche Trend arbeitet in dem Maße, wie die Armut steigt, daran, den Begriff der Armut zu relativieren, als ob nicht der Reichtum unserer Gesellschaften angesichts steigender Verelendung obszön ist, sondern das Sprechen über die Armut. So bleiben Armut und Reichtum als gesellschaftliche Phänomene auch im Bereich ihrer Messverfahren mit Werturteilen verbunden. Da heißt es beispielsweise nach der Feststellung, Deutschland sei »ein reiches Land «: »Soziale Sicherheit folgt künftig dem Paradigma, die Menschen zu befähigen, flexibel auf die Herausforderungen der Dienstleistungs- und Wissensgesellschaft zu reagieren « - also nicht mehr dem Paradigma »materieller Umverteilung « (Armutsbericht 2005). Die Armut ist also ein Problem der »Fähigkeiten « der Menschen, so dass es nicht mehr sehr weit zum Diskurs der selbstverschuldeten Armut des 18. und 19. Jahrhunderts ist.

Ähnlich ursachenverschleiernd verfährt der Armutsbericht in der Frage migranti- scher Armut: »Das höhere Risiko ausländischer Haushalte, auf Sozialleistungen angewiesen zu sein ", sei »vor allem auf höhere Arbeitslosigkeit infolge geringerer Bildungs- und Ausbildungsbeteiligung zurückzuführen ... Diese Defizite sind ... Ursache für ein besonderes Arbeitsmarktrisiko von Ausländerinnen und Ausländern « -- kein Wort von institutioneller Diskriminierung, die beispielsweise darin besteht, dass der Defizitblick bis heute die Potenziale von Migrantenkindern in Schule und Ausbildung brachliegen lässt, oder die sogenannten Drittstaatsangehörigen nach den Deutschen und den EUBürgern an dritter Stelle auf offene Stellen vermittelt werden, weshalb auch die Arbeitslosigkeit unter ihnen dreifach so groß ist. Diese Form von Erklärungen sowie die gegenwärtigen Debatten zur Parallelgesellschaft haben zum Teil den Charakter eines Entlastungsdiskurses, der der Mehrheitsgesellschaft erlaubt, ihrer Verantwortlichkeit aus dem Weg zu gehen. Die strukturelle Chancenlosigkeit migrantischer Lebenswelten in Zeiten verschärfter Deregulierung und des Abbaus sozialer Transferleistungen ist die soziale Seite der Migrationsmedaille.

\section{Soziale Aspekte der Arbeit islamischer Organisationen in Deutschland}

Dass die soziale Frage zu einem systematischen Tagesordnungspunkt islamischer Organisationen wird, wird wahrscheinlich noch lange auf sich warten lassen. Während beispielsweise die Caritas als Antwort auf die sogenannte Arbeiterfrage entstanden war, sind die islami-

\section{Vor Statistiken wird gewarnt}

Die verbreiteten Zahlen der Muslime in Deutschland halten einer Überprüfung nicht stand: Sie werden lediglich aufgrund der Herkunft ermittelt und sowohl von staatlichen Institutionen als auch in der Wissenschaft und selbstverständlich mit Vorliebe durch die islamischen Organisationen verwendet. So entstehen Zahlen von über drei Millionen und mehr Muslimen in Deutschland. Dass nicht alle Menschen beispielsweise aus der Türkei Muslime sind, möglicherweise zahlreiche Atheisten unter ihnen sind, dazu unterschiedlichste Formen von Religiosität (im Alevitentum gibt es unaufhörliche und berechtigte Debatten darüber, ob sie überhaupt dem Islam angehören) anzutreffen sind, sind so ermittelte Statistiken Unfug. Deren Absurdität wird deutlich, wenn wir einmal annähmen, dass wir als Wissenschaftler in einem nahöstlichen Land beginnen würden, alle Europäer im Orient als Christen zählen zu wollen - islamistische Organisationen, die im Orient so verfahren, gibt es tatsächlich.

Prof. Dr. Gazi Caglar

schen Organisationen in Deutschland sogar in der Atmosphäre modernster Arbeitsgesellschaft und ihrer strukturellen Krise noch weit davon entfernt, angemessene Antworten auf die Herausforderungen der sozialen Lage derjenigen zu entwickeln, die sie repräsentieren. Es ist bis heute ein erklärungswürdiges, keineswegs also selbstverständliches, hier lediglich erwähntes Phänomen, dass der neuere Islam in Deutschland zumindest in seiner Gemeindestruktur ein kulturelles Produkt der Arbeitsmigration ist, ohne ihre Rahmenbedingungen und die mit ihr verbundenen sozialen Zerwürfnisse zu reflektieren.

Können wir also eine gewisse »soziale Blindheit " für den Islam in Deutschland gegenüber den Problemlagen der modernen Arbeitsgesellschaft feststellen, so darf dies uns nicht dazu führen, die konkreten sozialen Aktivitäten islamischer Organisationen zu unterschlagen. Es soll lediglich auf das Defizit hinweisen, das sowohl dem Islam allgemein als auch spezifischen Formen in Deutschland eigen ist und darin besteht, bis heute keine spezifisch islamische Antwort auf die sozialen Herausforderungen des Kapitalismus »von unten « entwickelt zu haben. So haben sich die islamischen Organisationen in Deutschland von Anfang an, sozusagen jenseits der sozialen Frage in der Migrationsgesellschaft, um die religiösen und kulturellen Belange von »Gastarbeitern « gekümmert und vielfach deren Organisierungspotenziale für die Unterstützung verbundener islamischer Strömungen und Parteien in ihren Herkunftsländern verbraucht. Das hat sich zwar mit der zunehmenden Etablierung von Migrantinnen und Migranten seit Mitte der 80er Jahre des letzten Jahrhunderts verändert, ist aber bis heute nicht gänzlich verschwunden. Erst seit Beginn dieses Jahrhunderts lässt sich unter veränderten strukturellen Vorzeichen (Etablierung ethnischer Minderheiten in der Einwanderungsgesellschaft Deutschland; zunehmendes Verschwinden der Rückkehroption; das Vordringen der zweiten und dritten Generation in die Organisationen; verbreitete Islamophobie seit den Attentaten vom 11. September; vorsichtige Öffnung staatlicher Politik gegenüber »Integration « usw.) eine langsame, aber sichere Umorientierung islamischer Organisationen auf die hiesige Gesellschaft und ihre Herausforderungen feststellen, 
wobei sie gegenwärtig hauptsächlich mit Fragen der kulturellen und religiösen Anerkennung beschäftigt sind.

So haben bis heute islamische Organisationen, die selbst nicht nach dem Modell des »waqf « gegründet sind, sondern in ihrem Gros auf Spenden, Mitgliedsbeiträge und Sammlungen beim Freitagsgebet angewiesen sind, zwar dem Namen nach Vereine mit sozialem Auftrag gebildet, aber islamische soziale Dienstleistungen weder begrifflich noch praktisch mit Leben erfüllt. So führt die Türkisch-Islamische Union der Anstalt für Religion e. V. (DITIB) als größter muslimischer Dachverband in Deutschland in ihrer Internetpräsenz zwar die Rubrik »Integration und soziale Aufgaben ", versteht darunter aber neben Computerkursen und Folklore von der Bundesregierung finanzierte Integrations- und Orientierungskurse. Ebenso existieren Organisationen wie der »Islamische Wohlfahrtsverband « im Kontext des Verbandes der Islamischen Kulturzentren e. V. (VIKZ), zu dessen Aufgaben laut Satzung die
Wahrnehmung und Besetzung sozialer Aufgabenfelder.

\section{Die Moschee, spezielle Organi- sationen und die alevitischen Kulturvereine als soziale Orte}

Sowohl die Moscheen als auch die alevitischen Kulturvereine sind die zentralen Basisinstitutionen organisiert gelebter islamischer Alltäglichkeit in Deutschland, die zwar bei weitem nicht die Zahlen mobilisieren können, die sie als die Zahlen der jeweiligen Gläubigen angeben, dennoch als organisierte Formen ernst zu nehmen sind. Moscheen sind im Islam nicht nur Gebetsräume, noch weniger die alevitischen Kulturzentren. Vielmehr sind sie zugleich Orte der sozialen, kulturellen und politischen Kommunikation und des gegenseitigen solidarischen Beistandes. Die Integration in ein soziales Netzwerk charakterisiert sowohl die Moscheen als auch die alevitischen Kulturvereine. Sie geben einen Rückhalt, den man tagtäglich

\section{»In Moscheen und Kulturvereinen erfabren Muslime Gemeinschaft und Gemeinsamkeit, Dazugehörigkeit und Anerkennung «}

Gründung sozialer Einrichtungen wie Kindergärten, Altenheime, Jugendstätten etc. gehören. In der Praxis sind sie aber hauptsächlich über das Islamische Bildungswerk im Bildungsbereich aktiv. Im Kontext von Islamischer Gemeinschaft Milli Görüs e. V. (IGMG) gibt es ebenfalls Organisationen mit einem »Sozial « im Namen (Moslemisches Sozialwerk in Europa e. V., Muslimischer Sozialbund e. V.), aber sie beschäftigen sich hauptsächlich mit Bestattungswesen von Bestattungsfonds bis hin zu Bestattungshilfevereinen, die übrigens sowohl in allen sunnitischen, als auch schiitischen und alevitischen Richtungen weit verbreitet sind.

Wir können also festhalten, dass die Zentralverbände die Bedeutung sozialer Aufgaben in der modernen Gesellschaft theoretisch noch nicht begriffen und reflektiert haben. Und praktisch fehlen die Schritte in Richtung einer umfassenden auch außerhalb von Stress-Situationen erhält. Hier findet sozialer Bezug nicht nur mit Blick auf Problembewältigung, sondern vielmehr als ständiges Bedürfnis nach Kontakten, Interaktion, Rückmeldung, Zuwendung, Akzeptanz etc. statt. Im Kontext des spezifischen Belastungsbewältigungsprozesses der Migration übernehmen also religiöse Organisationen der Muslime soziale Unterstützungsfunktionen. Ihre Mitglieder bekommen die Chance, Gemeinschaft und Gemeinsamkeit zu erleben, erfahren Dazugehörigkeit und Anerkennung, erleben herkunftskulturelle Dimensionen, die in der Diaspora umso wichtiger werden. Die religiösen Inhalte liefern interpretative und deutende Unterstützung bei der Bewältigung von Diskriminierungserfahrungen.

In den Moscheen ergeben sich auch praktisch instrumentelle Beziehungen, die zur Bewältigung schwieriger beruflicher
Situationen und Arbeitslosigkeit dienen können. Niedrigschwellige Beratung, soziokulturelle Bildung, individuelle psychische Stabilisierung, Armenfürsorge und Hilfe für Obdachlose finden also hier statt. Moscheen und alevitische Kulturzentren, die hier gelebte Alltagskultur gegenseitigen Beistandes und der Begleitung in Krisensituationen (teilweise spontan organisierter Beistand, beispielsweise durch Sterbebegleitung) sind soziale Aspekte islamischen Lebens in Deutschland, die in Summen kaum berechnet werden könnten. Dieses Leben ist eine der Vielfalt: institutionalisierte und zentralistische Formen, Religion ohne Institution, traditionelle Formen des »Volksislams «, Patchwork-Religiosität, Formen neuer Religiösität - eine Vielfalt ist in fast jeder deutschen Stadt zu finden. Prozesse der Enttraditionalisierung und der permanenten Produktion von Tradition gehen dabei Hand in Hand.

Darüber hinaus gibt es auch anerkannte Träger Sozialer Arbeit, für die beispielhaft an dieser Stelle das Begegnungs- und Fortbildungszentrum muslimischer Frauen e. V. (BFmF) in Köln genannt werden soll, das als Selbsthilfeprojekt muslimischer Frauen in multikultureller Zusammensetzung gegründet wurde und ein umfassendes Bildungs- und Beratungsangebot bereithält. Als soziale Anlaufstelle mit Modellcharakter erfüllt das Zentrum wichtige soziale Aufgaben und ist als einzige muslimische Einrichtung der Sozialen Arbeit in Nordrhein-Westfalen anerkannt.

Projektarbeit ist jedoch die vorherrschende Existenzweise muslimischer und alevitischer Sozialer Arbeit. Dies hängt stark mit dem Wohlfahrtsmonopolismus in Deutschland zusammen, in den die zahlreichen muslimischen Trägerstrukturen durch eigene Professionalisierung und fremde Anerkennung die ersehnte Aufnahme noch nicht erreicht haben. So weisen sie noch einen großen Professionalisierungsrückstand aus, der sich aus Schwierigkeiten der Institutionalisierung einerseits und einem in Europa zumindest aus der Sicht der emanzipatorischen Sozialen Arbeit überholten Fürsorgeverständnis und einem korporatistischen Verständnis von Individuum und Gemeinschaft andererseits ergibt (»unsere Jugendlichen vor den Gefahren der Stadt schützen «, »Drogenabhängigkeit verhindern ", "auf den rechten Weg bringen « etc.). 


\section{Islamischer Wohlfahrtsverband}

Dies wird aber mittelfristig nicht so bleiben können. Die zahlreichen Bemühungen um Formulierung und Angebot professioneller sozialer Dienstleistungen, die Kämpfe zur Anerkennung als gleichberechtigte Partner mit den anderen religiösen Anbietern, die wachsende Bedeutung auch kulturell differenzierter Angebotsstrukturen, die zunehmende professionelle Ausbildung muslimischer Fachkräfte wird über kurz oder lang zur Entstehung eines oder mehrerer islamischer Wohlfahrtsverbände führen, wenn diese nicht willkürlich oder per Monopolstellung der bestehenden verhindert werden sollten.

Nun ist leicht zu verstehen, warum der Islam gegenwärtig sowohl auf Bundes- als auch Länderebene immer mehr Anerkennung durch die offizielle Politik erfährt. Neben anderen Faktoren spielt dabei sicherlich der Umstand eine große Rolle, dass in Zeiten zunehmender sozialer Zerklüftung der Gesellschaft die islamischen
Organisationen eine herausragende Rolle bei der sozialen Kontrolle und gesellschaftlichen Disziplinierung von Migrantinnen und Migranten im Allgemeinen und der ordnungspolitischen Kontrolle und Isolierung islamistischer Tendenzen im Besonderen spielen. Diesen Zielsetzungen entsprechen einerseits die ideologisch belasteten, kulturalistischen Reproduktionen aktueller Diskurse und andererseits die symbolische politische Anerkennungspraxis in Form beispielsweise von Islamkonferenzen und Islamunterricht. Dass gesamtgesellschaftlich über die Debatten zur Parallelgesellschaft und der Gefahr des islamistischen Terrorismus auch Identitätsstiftung für die Mehrheitsgesellschaft, die Hinnahme enormen sozialen Abbaus seit der Wiedervereinigung sowie ein enormer Ausbau des Sicherheitsstaates organisiert werden, muss wohl als bittere Ironie verstanden werden.

Der Islam versucht gegenwärtig, diese günstige Situation zu nutzen und aus der soziokulturellen Randstellung, die teilweise Ergebnis der sozioökonomischen Marginalisierung von Migrantinnen und Migranten in Deutschland ist, herauszukommen. Die neuerliche Tendenz zum Bau repräsentativer Moscheen ist lediglich ein Beispiel für diesen Weg in die Mitte der Gesellschaft, die sie bisher hauptsächlich negativ belegt hat. Dabei erhebt er Anspruch auf die Gleichbehandlung mit anderen Religionen, möchte an ihren Privilegien teilhaben.

Nun werden drei Viertel der sozialen Dienstleistungen in Deutschland von den Wohlfahrtsverbänden organisiert. Insbesondere gilt dies für Kindergärten, ambulante Dienste, Alten- und Pflegeheimen und fast der Hälfte der Krankenhäuser. $\mathrm{Zu}$ ihnen gehören auch die Einrichtungen mit religiöser Orientierung von Caritas, Diakonie und der Zentralen Wohlfahrtsstelle der Juden in Deutschland. Die Anerkennungskämpfe der Muslime und Aleviten werden vor dieser Form religiös orientierter Wohlfahrt mit Sicherheit nicht haltmachen.

\section{Soziale Aspekte des Islam als Religion}

Die Muslime in Deutschland haben angesichts der Situation von Migrantinnen und Migranten allen Grund, sich der sozialen Frage anzunehmen. Der Islam in Deutschland trägt bis heute deutliche Spuren sozial marginalisierter Lebenswelten. Seine religiös motivierte Fürsorge ist kaum geeignet, die sozialen Folgen der Marginalisierung migrantischer Lebenswelten in der spätkapitalistischen Gesellschaft aufzufangen. Dabei verdeckt die Islamophobie die realen sozialen und kulturellen Problemlagen, die sich gerade durch den Rückzug des Sozialund Rechtsstaates verschärfen. Dennoch bietet der Islam für gläubige Muslime Anknüpfungspunkte, die einer Anpassung an die Erfordernisse der Gegenwart harren.

Die islamische Religion ist ihrem Selbstverständnis nach die letzte Kette der großen Abrahimitischen Religionen. Sie nimmt die biblischen Propheten und Prophetien an und verehrt sie. Sie lässt aber, streng genommen, keine Individuen, Gruppen oder Klassen als Vermittler zwischen Gott und den Menschen zu. Alle Menschen sind in ihrem Bezug zu Gott gleich, egal welche Hautfarbe, Abstammung oder Herkunft sie haben. Nur Frömmigkeit erkennt der Koran als privilegierender Unterschied zwischen Menschen an. Zudem werden die Gläubigen immer wieder aufgerufen, gerecht $\mathrm{zu}$ sein. Betont wird die Rolle des Gebens an Menschen, die aus verschiedenen Gründen sich selbst nicht versorgen können: Als frommste Tat gilt die Sättigung der Armen, die Unterbringung der Obdachlosen, die Ausbildung der Bildungsfernen, die Gründung von Institutionen, die Bedürftigen Unterhalt geben. Schließlich ist es trotz der Betonung der Eigenverantwortlichkeit des Individuums für sein Handeln ausschlaggebendes Ziel, eine ideale Gemeinde einzurichten, in der der Einzelne mit der Gemeinschaft verwoben ist.
Gebet und soziale Abgabe (Spende) sind gleichermaßen wichtig, die soziale Dimension ist von zentraler Bedeutung. Die Armenabgabe (»zakat «) soll den Gläubigen »reinigen «, denn alles Eigentum bleibt unrein, wenn diese obligatorische Steuer von jährlich 2,5 Prozent (manchen zufolge bis zu 10 Prozent) nicht bezahlt wird. Die obligatorische Spende ist also als eine Pflichtabgabe, ein festes Fundament wie der Glaube an Gott. Man könnte deuten, dass umgekehrt die zum Empfang Berechtigten keine Almosenempfänger sind, sondern ein Recht darauf haben. Zusätzlich kennt der Islam die Institution der »sadaka " als Form freiwilliger Spende oder Almosen und der »heba « als Geschenk.

Neben diesen transzendentalen Quellen gibt es noch die Institution der wohltätigen Stiftung, der »waqf «, mit ihren vielen Arten. Öffentliche Stiftungen (»waqf hayri «) wie auch Familienstiftungen (»waqf ahli «) erfüllten in vorkapitalistischen Gesellschaften des Nahen Ostens eine Vielzahl von sozialen Aufgaben, die von der Armen- und Krankenfürsorge über die Finanzierung eines breiten Spektrums an religiösen und Bildungseinrichtungen bis zu allgemeinen Infrastrukturaufgaben reichten. Die »waqf « als geschichtliche islamische Form von Stiftungen mit sozialem Inhalt ist in Deutschland bisher nicht bekannt, obwohl sie in ihrer Vielfalt bis heute im Nahen und mittleren Osten enorm verbreitet ist. Ebenso ist kaum etwas zu spüren davon, dass die Institution der » zakat « und » heba « organisiert und systematisch dazu genutzt werden, das allgemeine soziale Elend in Deutschland oder nur unter Migrantinnen und Migranten zu lindern, denn der Islam kennt auch keine Glaubensgrenze bei der Vergabe der Pflichtabgabe.

Prof. Dr. Gazi Caglar 